\title{
Forme fruste of isolated right ventricular endomyocardial fibrosis: a case report
}

\author{
Matthew Bunte ${ }^{1}$, Kenneth Liao ${ }^{2}$, J Carlos Manivel ${ }^{3}$ and Emil Missov ${ }^{1 *}$
}

\begin{abstract}
Introduction: Endomyocardial fibrosis is a neglected tropical disease of unknown etiology and poor prognosis. It is endemic of tropical climates where it is the most common cause of restrictive cardiomyopathy in the second and fourth decades of life. A forme fruste of the disease is thought to be present in temperate climates where the diagnosis remains exceedingly rare.
\end{abstract}

Case presentation: We describe a case of isolated right ventricular endomyocardial fibrosis in a 27-year-old Caucasian man from a temperate climate who presented initially with frank hemoptysis and pulmonary thromboembolic disease. We further describe the approach utilized in the diagnosis, the surgical treatment and the outcome of the disease.

Conclusions: We conclude that endomyocardial fibrosis should be included in the differential diagnosis of apical cardiomyopathies in patients from temperate climates.

Keywords: Endomyocardial fibrosis, Venous thromboembolism, Transesophageal echocardiography, Cardiac magnetic resonance imaging

\section{Introduction}

Endomyocardial fibrosis is the most common cause of restrictive cardiomyopathy in tropical climates [1]. It is characterized by idiopathic fibrosis of the endomyocardium and is frequently associated with thromboembolic disease, arrhythmias and complications of chronic heart failure $[2,3]$. A forme fruste of the disease is thought to be present in temperate climates where the diagnosis remains exceedingly rare [4].

\section{Case presentation}

A 27-year-old Caucasian man presented with frank hemoptysis preceded by a two-month history of shortness of breath and non-productive cough. Prior to this subacute illness, he was healthy, had no travel history, and was a long-distance endurance athlete. An intravenouslycontrasted computerized tomogram of the chest revealed bilateral pulmonary emboli and a filling defect within his right ventricle. Duplex lower extremity ultrasonography was negative for deep vein thrombosis. Transesophageal echocardiography revealed a mass at his right ventricular

\footnotetext{
*Correspondence: misso001@umn.edu

'Division of Cardiology, University of Minnesota Medical Center, Minneapolis, MN 55455, USA

Full list of author information is available at the end of the article
}

apex spanning proximally as pedunculated extensions through the tricuspid valve. Gadolinium-enhanced cardiac magnetic resonance imaging (MRI) confirmed a complex mass obliterating his right ventricular apex, insinuated between the chordae tendineae of the tricuspid valve and terminating in lobular stalks (Additional file 1). Surgical excision was recommended as the mass was felt to represent high risk for recurrent embolic events. The gross appearance of the mass was smooth, yellow and lobulated (Figure 1). Two distinct heads projected through the tricuspid valve and extensively insinuated between the chordae tendineae, requiring right ventricular endocardectomy with excision and replacement of the tricuspid valve. On histopathological examination, normal myocardium was covered by thickened fibrous endocardium with dense collagen, rare non-specific inflammatory cells with no eosinophils and a layer of arterioles, small veins and capillaries at the interphase with the myocardium (Figure 2). These findings are pathognomonic of endomyocardial fibrosis.

Anticoagulation therapy was initiated post-operatively. Extensive work-up for coagulopathy was negative. Peripheral blood and bone marrow biopsy were normal; in particular, the percentage of eosinophils was normal at $2 \%$. Cytogenetic study of the bone marrow showed no evidence of a malignant process. Other causes of endomyocardial 


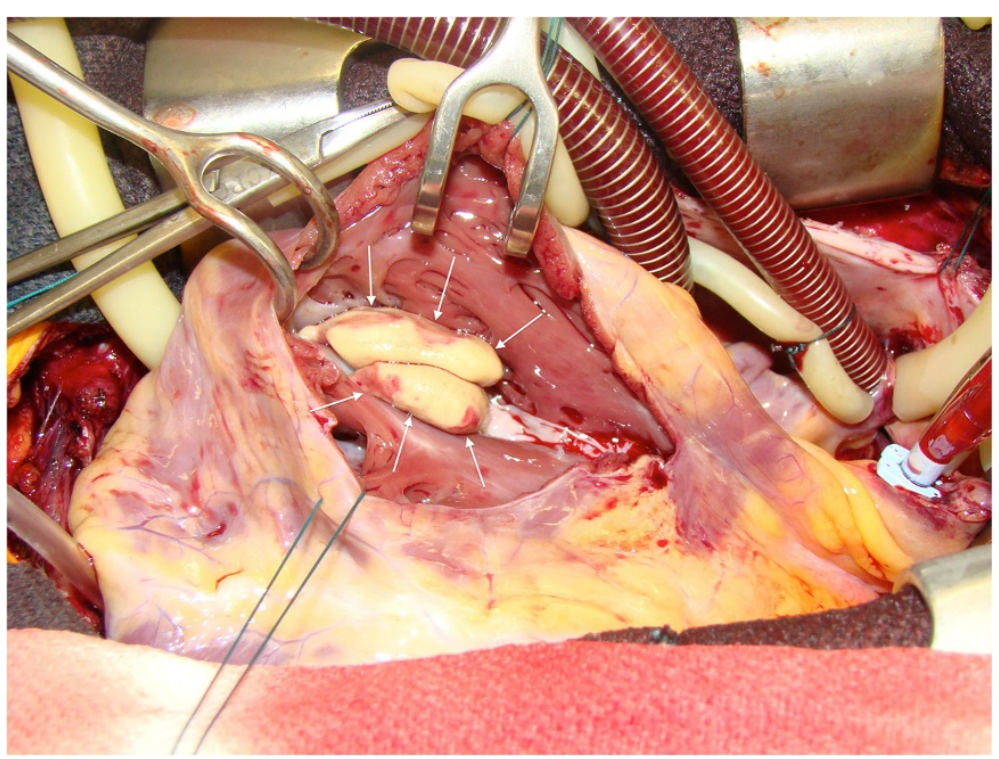

Figure 1 Surgical findings. A large, smooth, yellow mass (arrows) with lobulated heads was found in the right ventricular cavity following ventriculotomy.

fibrosis, including parasitic infections, carcinoid tumor and systemic diseases, such as Churg Strauss syndrome, periarteritis nodosa and Behçet syndrome, were excluded.

\section{Discussion}

The etiology and pathogenesis of endomyocardial fibrosis remain poorly understood. Several mechanisms have been postulated, including environmental and genetic factors, nutritional deficiencies, parasitic infections and

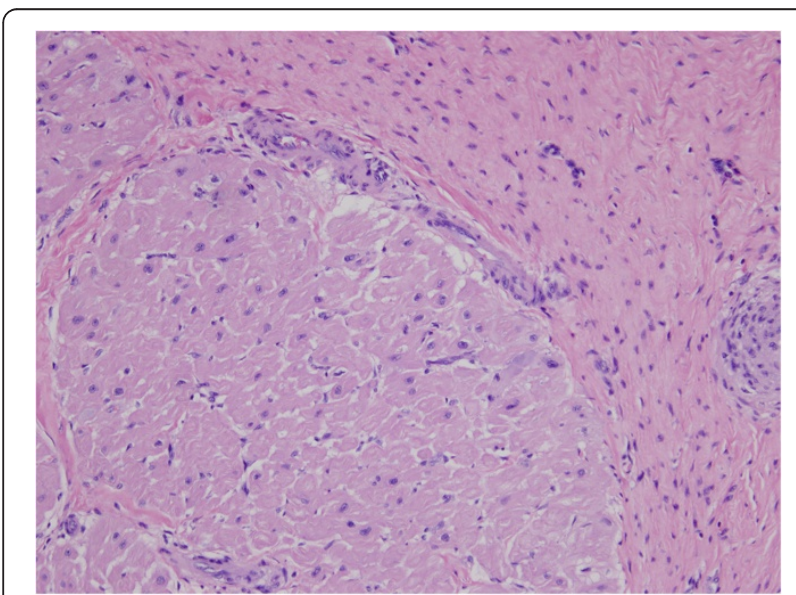

Figure 2 Histopathological findings. Markedly thickened fibrous endocardium (upper right) overlies normal myocardium (lower left). A layer of arterioles, venules and capillaries is seen at the interphase between the myocardium and thickened endocardium. Eosinophils are not present. (Hematoxylin-eosin stain; original magnification, $\times 400$ ). eosinophilia. Recent studies have demonstrated antibodies against myocardial proteins and a high incidence of cardiotropic infective agents like cytomegalovirus, Epstein-Barr virus and Toxoplasma gondii in affected patients, suggesting a role for inflammation [5]. The prevalence of endomyocardial fibrosis peaks in the second and fourth decades of life with a male preponderance among all age groups. Approximately 55\% of patients diagnosed with endomyocardial fibrosis have biventricular involvement and $17 \%$ have left-sided disease. The prevalence of isolated right ventricular endomyocardial fibrosis is $28 \%$ [1].

The diagnosis of endomyocardial fibrosis relies on echocardiographic criteria. The most salient features are a mass associated with one or both ventricles, typically the apices, and mural thrombus on the endocardial surface [3,4]. Cardiac MRI is a technique that provides additional information about ventricular hypertrophy, myocardial fibrosis, intracavitary masses and mural thrombosis [6]. Histopathological examination in endomyocardial fibrosis typically describes a thickened endocardium with elastic fibers and dense collagen, non-specific inflammatory cells, and a layer of arterioles, small veins and capillaries [5].

\section{Conclusions}

Endomyocardial fibrosis is rare in temperate climates and should be included in the differential diagnosis of apical cardiomyopathies. Echocardiography and cardiac MRI each provide important information in the diagnosis of endomyocardial fibrosis; histopathological examination remains essential for the final diagnosis. 


\section{Consent}

Written informed consent was obtained from the patient for publication of this Case Report and any accompanying images. A copy of the written consent is available for review by the Editor-in-Chief of this journal.

\section{Additional file}

Additional file 1: Cine magnetic resonance imaging findings. Cine cardiac magnetic resonance imaging study in the four-chamber view shows a large intracavitary space occupying mass in the right ventricle. The mass is polypoid and lobulated, and marginally mobile. Note the concordance with the surgical findings in Figure 1.

\section{Abbreviation}

MRI: Cardiac magnetic resonance imaging.

\section{Competing interests}

The authors declare that they have no competing interests.

\section{Authors' contributions}

$\mathrm{MB}$ was a major contributor in writing the manuscript. $\mathrm{KL}$ obtained the surgical specimen and was a major contributor in writing the manuscript. JCM performed the histological examination of the surgical specimen. EM performed the transesophageal examination, image analysis and interpretation, and was a major contributor in writing the manuscript. All authors read and approved the final manuscript.

\section{Author details}

'Division of Cardiology, University of Minnesota Medical Center, Minneapolis, MN 55455, USA. ${ }^{2}$ Division of Cardiothoracic Surgery, University of Minnesota Medical Center, Minneapolis, MN 55455, USA. ${ }^{3}$ Department of Laboratory Medicine and Pathology, University of Minnesota Medical Center, Minneapolis, MN 55455, USA.

Received: 4 May 2013 Accepted: 10 January 2014

Published: 10 March 2014

\section{References}

1. Mocumbi AO, Ferreira MB, Sidi D, Yacoub MH: A population study of endomyocardial fibrosis in a rural area of Mozambique. $N$ Engl J Med 2008, 359:43-49.

2. Ribeiro PA, Muthusamy R, Duran CM: Right-sided endomyocardial fibrosis with recurrent pulmonary emboli leading to irreversible pulmonary hypertension. Br Heart J 1992, 68:326-329.

3. Hassan WM, Fawzy ME, Al Helaly S, Hegazy H, Malik S: Pitfalls in diagnosis and clinical, echocardiographic, and hemodynamic findings in endomyocardial fibrosis. Chest 2005, 128:3985-3992.

4. Goodwin JF: Endomyocardial disease-clinical features. Postgrad Med J 1983, 59:154-156.

5. Iglezias SD, Benvenuti LA, Calabrese F, Salemi VM, Silva AM, Carturan E, de Oliveira SA, Thiene G, De Brito T: Endomyocardial fibrosis: pathological and molecular findings of surgically resected ventricular endomyocardium. Virchows Arch 2008, 453:233-241.

6. Qureshi N, Amin F, Chatterjee D, Prasad S, Al-Obaidi M, Missouris C: MR imaging of endomyocardial fibrosis (EMF). Int J Cardiol 2011, 149:e36-37.

doi:10.1186/1752-1947-8-94

Cite this article as: Bunte et al:: Forme fruste of isolated right ventricular endomyocardial fibrosis: a case report. Journal of Medical Case Reports 2014 8:94.

\section{Submit your next manuscript to BioMed Central and take full advantage of:}

- Convenient online submission

- Thorough peer review

- No space constraints or color figure charges

- Immediate publication on acceptance

- Inclusion in PubMed, CAS, Scopus and Google Scholar

- Research which is freely available for redistribution 